\title{
End-Stage Renal Disease After Renal Surgery in Patients with Normal Preoperative Kidney Function: Balancing Surgical Strategy and Individual Disorders at Baseline
}

\author{
Umberto Capitanio $^{a, b, *}$, Alessandro Larcher $^{a, b}$, Carlo Terrone $^{c}$, Alessandro Antonelli $^{d}$, \\ Alessandro Volpe ${ }^{c}$, Cristian Fiori $^{f}$, Maria Furlan $^{d}$, Federico Deho ${ }^{a, b}$, Andrea Minervini $^{e}$, \\ Sergio Serni ${ }^{e}$, Francesco Porpiglia $^{f}$, Francesco Trevisani $^{b, g}$, Andrea Salonia $^{a, b}$, Marco Carini $^{e}$, \\ Claudio Simeone ${ }^{d}$ Francesco Montorsi $^{a, b}$, Roberto Bertini $^{a, b}$
}

\begin{abstract}
${ }^{\mathrm{a}}$ Unit of Urology, University Vita-Salute, San Raffaele Scientific Institute, Milan, Italy; ${ }^{\mathrm{b}}$ Division of Oncology/Unit of Urology; URI; IRCCS Ospedale San Raffaele, Milan, Italy; ${ }^{\mathrm{C}}$ Department of Urology, University of Piemonte Orientale, Novara, Italy; ${ }^{\mathrm{d}}$ Department of Urology, Universita' degli studi e Spedali Civili di Brescia, Brescia, Italy; ${ }^{\mathrm{e}}$ Department of Urology, Clinica Urologica I, Azienda Ospedaliera Universitaria Careggi, Università degli studi di Firenze, Florence, Italy; ${ }^{\mathrm{f}}$ Department of Urology, AOU San Luigi Gonzaga, Università degli studi di Torino, Orbassano, Italy; ${ }^{\mathrm{g}}$ Unit of Nephrology, San Raffaele Scientific Institute, Milan, Italy
\end{abstract}

\section{Article info}

Article history:

Accepted March 16, 2016

Associate Editor:

Giacomo Novara

Keywords:

Kidney cancer

Partial nephrectomy

Radical nephrectomy

Nephron-sparing surgery

End-stage renal disease

Dialysis

\section{EU $\star A C M E$}

www.eu-acme.org/

europeanurology

Please visit www.eu-acme.org/

europeanurology to read and

answer questions on-line.

The EU-ACME credits will

then be attributed

automatically.

\begin{abstract}
Although nephron-sparing surgery (NSS) has demonstrated benefit in terms of renal function preservation, it is unclear whether NSS might also decrease the risk of endstage renal disease (ESRD) relative to radical nephrectomy (RN). In the current paper, we aimed to report the rate and the predictors of ESRD after surgery, accounting for detailed individual baseline characteristics and comorbidities. A multi-institutional collaboration among five European tertiary care centers allowed study of 2027 patients with normal preoperative renal function and a clinically localized T1abNOM0 renal mass. Cox regression analyses were used to predict the risk of ESRD (defined as the onset of a postoperative estimated glomerular filtration rate $<15 \mathrm{ml} / \mathrm{min}$ per $1.73 \mathrm{~m}^{2}$ ) after adjusting for the individual baseline risk of developing chronic kidney disease. Univariable ESRD rates at 5 and $10 \mathrm{yr}$ of follow-up were virtually equivalent for patients who underwent NSS (1.5\% and $2.5 \%$, respectively) versus RN $(1.9 \%$ and $2.7 \%$, respectively; hazard ratio [HR]: 0.8; 95\% confidence interval [CI], 0.4-1.6). However, diabetes, smoking, uncontrolled hypertension, and other comorbidities were consistently more frequent in the NSS group relative to their RN counterparts. After adjusting for detailed baseline individual characteristics, NSS was shown to have an independent protective effect relative to RN (HR: $0.4 ; 95 \% \mathrm{CI}, 0.2-0.8 ; p=0.02$ ) at multivariable analyses. Patient summary: After accounting for individual baseline characteristics, such as age, diabetes, uncontrolled hypertension, or other comorbidities, partial nephrectomy independently protects against end-stage renal disease and the consequent need for dialysis relative to radical nephrectomy.
\end{abstract}

(C) 2016 European Association of Urology. Published by Elsevier B.V. All rights reserved. 
If technically feasible, nephron-sparing surgery (NSS) represents the standard of care for patients diagnosed with a clinically localized renal mass [1], mainly due to a demonstrated benefit in terms of preservation of renal function [2]. In a recent subanalysis of 514 patients included in the European Organization for Research and Treatment of Cancer (EORTC) randomized trial 30904, the incidence of end-stage renal disease (ESRD) was nearly identical for patients treated with either NSS or radical nephrectomy (RN) [2]. In the current paper, we aimed to report the rate and the predictors of ESRD after renal surgery. To limit the inherent risk of bias, we investigated a large multiinstitutional data set that allowed adjustment for a detailed panel of intrinsic confounders such as uncontrolled hypertension, diabetes, body mass index (BMI), and other comorbidities.

The current study relied on a collaborative database collected from five European tertiary care centers. Patients with a primary diagnosis of nonmetastatic clinical T1 unilateral kidney cancer without a baseline condition of chronic kidney disease who were treated with NSS or RN between 1984 and 2010 were included. The outcome of the study was ESRD, defined as the onset of a postoperative estimated glomerular filtration rate (GFR) $<15 \mathrm{ml} / \mathrm{min}$ per $1.73 \mathrm{~m}^{2}$. GFR was calculated by the Chronic Kidney Disease Epidemiology Collaboration formula in younger patients (aged $<70 \mathrm{yr}$ ) and by the Berlin Initiative Study formula in older patients (aged $\geq 70 \mathrm{yr}$ ) [3]. We included the following covariates: age, year of surgery, preoperative GFR, tumor size, hypertension (none, uncontrolled, or controlled by medical therapy), diabetes, baseline Charlson comorbidity index (CCI), BMI, and smoking status (no, yes, or former). First, descriptive statistics were reported. Second, multivariable Cox regression analyses were used to assess the impact of surgery type (NSS vS RN) on ESRD after adjustment for all available covariates. Finally, multivariable Cox regression coefficients were used to plot the covariate-adjusted ESRD rates according to different patient subgroups.

Overall, 2027 patients were included in the study (Table 1). Patients were treated with NSS $(65.8 \%$, $n=1334$ ) or RN (34.2\%, $n=693)$. Patients treated with NSS were more frequently diagnosed with concomitant comorbidities ( $\mathrm{CCI} \geq 0 ; 55 \%$ vs $40 \% ; p<0.001$ ), uncontrolled hypertension ( $21 \%$ vs $13 \% ; p<0.001$ ), diabetes ( $12 \%$ vs $7 \%$; $p<0.001$ ), and smaller tumor (32 vs $50 \mathrm{~mm} ; p<0.001$ ) (Table 1). Mean follow-up was 72 mo. Supplementary Figure 1 depicts ESRD events according to treatment type. Median time to ESRD was 45 mo (interquartile range: 19-106 mo). Unadjusted ESRD rates at 5 and $10 \mathrm{yr}$ of followup were virtually equivalent between patients who underwent NSS (1.5\% and $2.5 \%$, respectively) versus RN (1.9\% and

Table 1 - Clinical characteristics of 2027 patients with cT1NOMO renal tumor with normal renal function before surgery and treated with either nephron-sparing surgery or radical nephrectomy, stratified by treatment delivery

\begin{tabular}{|c|c|c|c|}
\hline Variable & NSS $(n=1334,65.8 \%)$ & $\mathrm{RN}(n=693,34.2 \%)$ & $p$ value \\
\hline \multicolumn{4}{|l|}{ Clinical characteristics } \\
\hline Age,yr, median (IQR) & $61(51-69)$ & $61(52-69)$ & 0.4 \\
\hline Gender, \% & & & 0.9 \\
\hline Male & 66.0 & 66.2 & \\
\hline Female & 34.0 & 33.8 & \\
\hline eGFR before surgery, $\mathrm{ml} / \mathrm{min} / 1.73 \mathrm{~m}^{2}$, median (IQR) & $86(82-98)$ & $85(85-93)$ & 0.002 \\
\hline Body mass index, median (IQR) & $25.7(23.8-27.9)$ & $25.8(23.8-27.9)$ & 0.7 \\
\hline Smoking status, \% & & & $<0.001$ \\
\hline No & 59.2 & 53.1 & \\
\hline Smoker & 27.3 & 22.6 & \\
\hline Former & 13.6 & 19.8 & \\
\hline Diabetes, \% & 12.5 & 7.4 & $<0.001$ \\
\hline Hypertension, \% & & & $<0.001$ \\
\hline No & 56.5 & 67.2 & \\
\hline Uncontrolled & 21.1 & 13.3 & \\
\hline Controlled by therapy & 22.4 & 19.5 & \\
\hline Charlson comorbidity index, \% & & & $<0.001$ \\
\hline 0 & 45.3 & 60.0 & \\
\hline 1 & 16.2 & 18.2 & \\
\hline$>1$ & 38.6 & 21.7 & \\
\hline Clinical tumor size, median (IQR) & $3.2(2.5-4.0)$ & $5.0(3.7-6.0)$ & $<0.001$ \\
\hline \multicolumn{4}{|l|}{ Surgical characteristics } \\
\hline Ischemia time, min, median (IQR) & $11(0-18)$ & NA & NA \\
\hline \multicolumn{4}{|l|}{ Type of ischemia, \% } \\
\hline No ischemia & 32.4 & & \\
\hline Warm ischemia & 66.9 & NA & NA \\
\hline Cold ischemia & 0.8 & & \\
\hline Open, $n$ (\%) & $889(66.6)$ & $615(88.7)$ & $<0.001$ \\
\hline Laparoscopic, $n(\%)$ & $315(23.6)$ & $78(11.7)$ & \\
\hline Robotic, $n(\%)$ & $130(9.7)$ & $0(0)$ & \\
\hline
\end{tabular}


$2.7 \%$, respectively; $p=0.5$; hazard ratio [HR]: $0.8 ; 95 \%$ confidence interval $[\mathrm{CI}], 0.4-1.6)$ (Supplementary Fig. 1). Age $(p<0.001 ; \mathrm{HR}: 1.1 ; 95 \% \mathrm{CI}, 1.1-1.1)$, presence of diabetes ( $p=0.002$; HR: $3.4 ; 95 \% \mathrm{CI}, 1.5-7.4)$, uncontrolled hypertension ( $p<0.001$; HR: $4.1 ; 95 \% \mathrm{CI}, 2.1-8.2)$, and $\mathrm{CCI}$ ( $>1$ vs $0 ; p=0.004$; HR: $3.4 ; 95 \% \mathrm{CI}, 1.5-7.8$ ) were associated with ESRD risk (Supplementary Table 1). At multivariable analyses, after adjusting for all detailed baseline individual characteristics, NSS showed an independent protective effect against ESRD relative to RN (HR: $0.4 ; 95 \% \mathrm{CI}, 0.2-0.8 ; p=0.02$ ) (Supplementary Table 1). Figure 1 shows the multivariate-derived ESRD cumulative rates according to treatment type, patient age, diabetes, and hypertension, which, alongside year of surgery, were the most informative predictors of ESRD (Supplementary Table 1).

ESRD is a life-threatening condition [4]. In young and healthy persons, the estimated risk of ESRD at $15 \mathrm{yr}$ is $0.04 \%$ (95\% CI, 0.008-0.09), reaching a peak of $0.3 \%$ (95\% CI, $0.2-$ 0.4 ) in healthy kidney donors [5]. Due to older age, high prevalence of associated comorbidities, and reduced
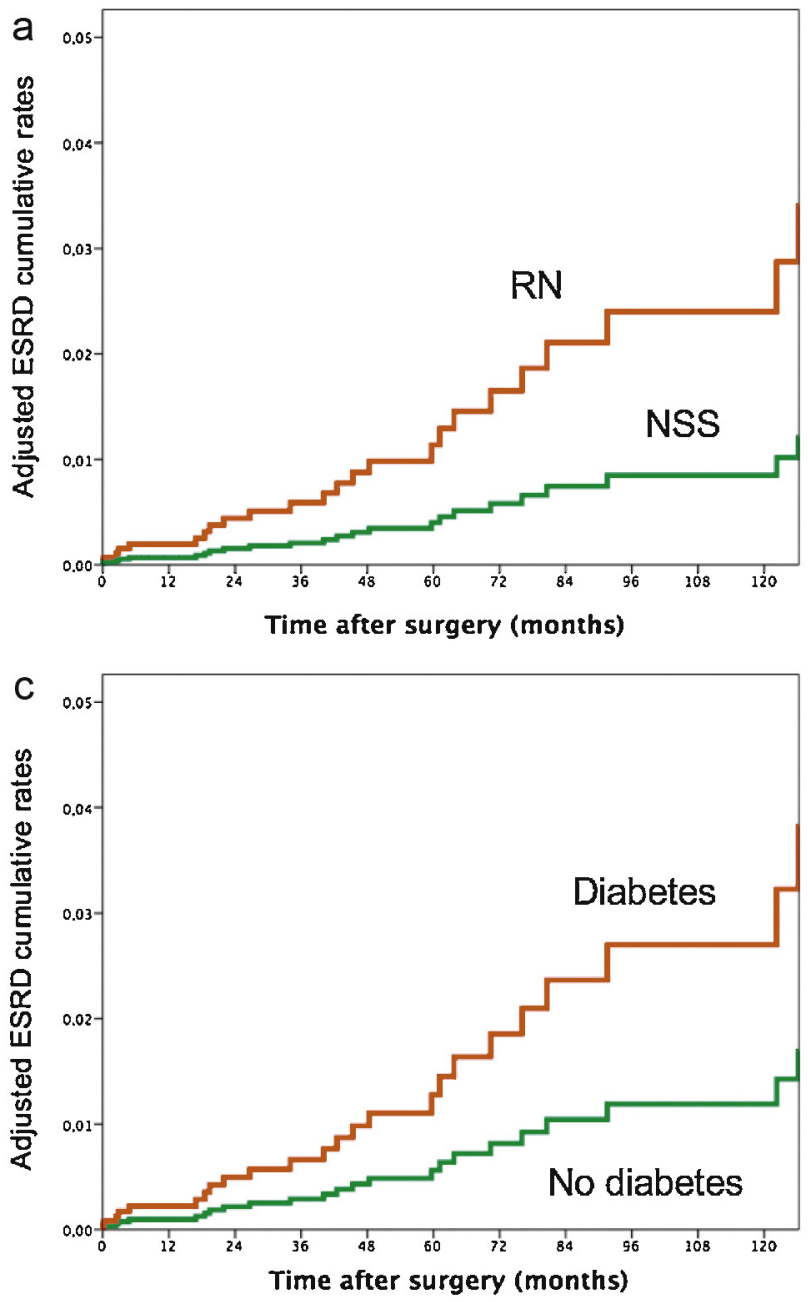

number of nephrons, ESRD risk after surgery for renal cancer is significantly more common. In such settings, ESRD has been already been prospectively reported in the EORTC trial 30904, showing rates very similar to the current report (1.5-1.6\% at a median follow-up of $6.7 \mathrm{yr}$ ) and demonstrating no difference in terms of ESRD rates between patients treated with NSS and RN $(-0.1 \%, 95 \% \mathrm{CI},-2.2$ to 2.1$)$ [2]. Although many limitations affected the original trial (eg, limited recruitment, crossover treatment, and lack of detailed comorbidity data of the patients), the EORTC trial suggested that ESRD might be related to intrinsic factors (eg medical conditions such as diabetes) that cannot be amended by the type of surgical technique delivered. That said, the current findings cannot be completely compared with the EORTC trial 30904. The randomized study included patients treated before 2003, some with preoperative renal function impairment (roughly 10\%) and with limited surgical experience in terms of NSS techniques and outcomes. The current series included patients who were treated more recently, who had normal preoperative renal function (in all patients treated), and who did not have hilar
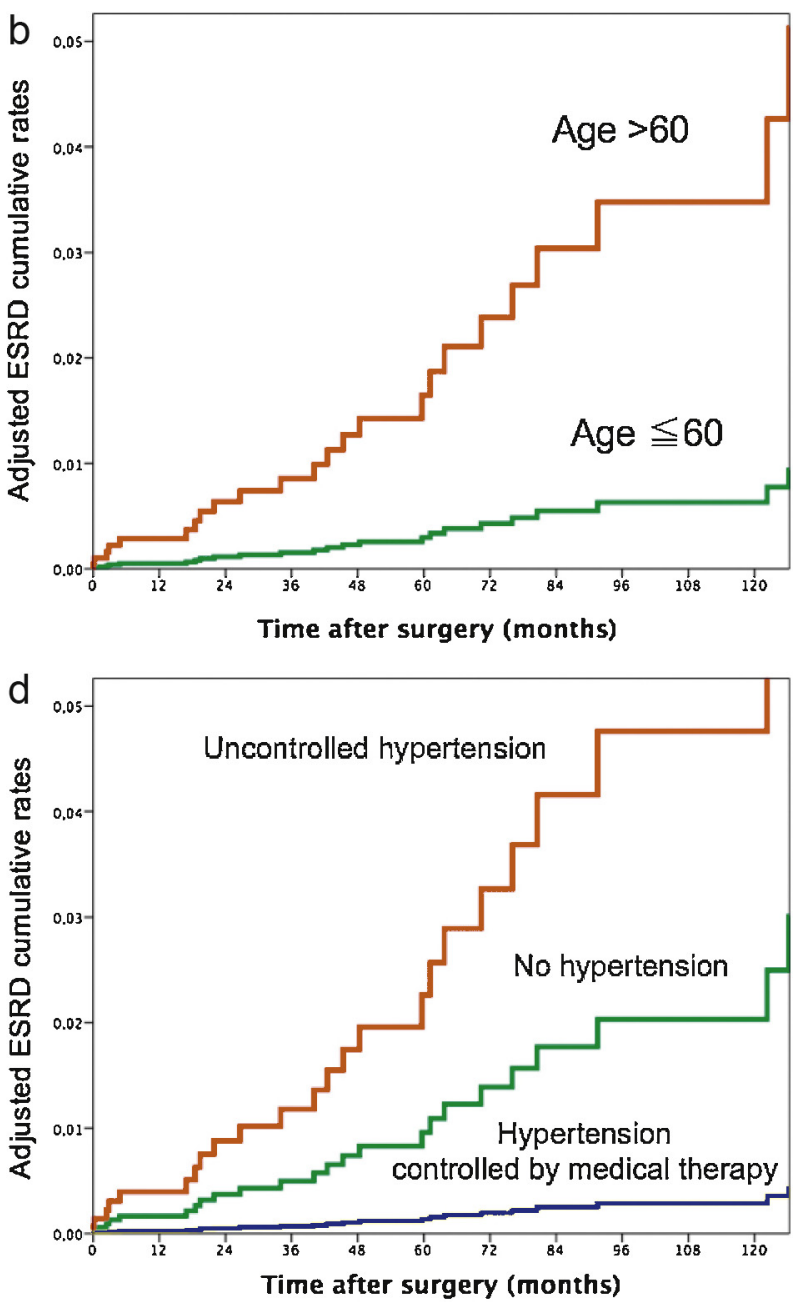

Fig. 1 - Adjusted ESRD cumulative rates stratified by (a) treatment delivery (NSS vs RN), (b) patient age, (c) presence of diabetes, and (d) hypertension (none, controlled by medical therapy, or uncontrolled). Data are corrected for age, year of surgery, body mass index, clinical tumor size, hypertension, preoperative estimated glomerular filtration rate, Charlson comorbidity index, diabetes, and smoking status. ESRD = end-stage renal disease; NSS = nephron-sparing surgery; $\mathrm{RN}$ = radical nephrectomy . 
clamping or who had limited (median: $11 \mathrm{~min}$ ) clamping (Table 1 ). Those main differences may explain the discordance between the benefit we found in the NSS group and the EORTC trial findings. In addition, Lin et al reported similar findings in a recent nationwide population-based study, although they could not adjust their findings for important determinants of chronic kidney disease, such as the presence of uncontrolled hypertension, BMI, and smoking status [6]. Finally, Yap et al recently anticipated a beneficial effect of NSS in decreasing the risk of ESRD (HR: $0.44 ; 95 \% \mathrm{CI}, 0.25-0.75)$, although they could not adjust the results for tumor characteristics (eg, tumor size, TNM), which are main determinants for surgical indication (NSS vs RN) and survival outcomes [7].

The current report introduces key findings. Considering the important causes of ESRD, such as diabetes, uncontrolled hypertension, and age, NSS appears to decrease the probability of ESRD after surgery. An even greater benefit is possible with the novel surgical techniques of no clamp or selective clamping, which were recently reported [1]. As already verified by others [8], baseline medical conditions that may produce renal functional impairment remain the key and most informative causes of renal failure, regardless of all surgical efforts to preserve nephrons [9]. However, our findings also corroborate the beneficial effect of NSS for the consequences of a baseline medical condition favoring chronic kidney disease [9].

The current study has several strengths, including the multi-institutional design, the relatively long follow-up, and the inclusion of patients without a condition of baseline chronic kidney disease. However, despite its appeal and uniqueness, the current study is not devoid of limitations, mainly due to the retrospective design of the study, which cannot exclude the presence of residual confounders (eg, proteinuria at diagnosis).

In conclusion, roughly $2 \%$ of the patients with normal estimated GFR before kidney surgery will develop ESRD in the first $10 \mathrm{yr}$ of follow-up. In addition to the already known protective benefits in terms of cardiovascular events and renal function preservation, NSS seems to be associated with a lower risk of ESRD relative to RN. Nonetheless, individual risk factors inherent at baseline (especially age, diabetes, and uncontrolled hypertension) appear to be crucial predictors of ESRD regardless of the treatment delivered.

Author contributions: Umberto Capitanio had full access to all the data in the study and takes responsibility for the integrity of the data and the accuracy of the data analysis.

Study concept and design: Capitanio, Larcher, Terrone, Antonelli, Volpe, Fiori, Furlan, Dehò, Minervini, Serni, Porpiglia, Trevisani, Salonia, Carini, Simeone, Montorsi, Bertini.

Acquisition of data: Capitanio, Larcher, Terrone, Antonelli, Volpe, Fiori, Furlan, Dehò, Minervini, Serni, Porpiglia, Trevisani, Salonia, Carini, Simeone, Montorsi, Bertini.

Analysis and interpretation of data: Capitanio, Larcher.

Drafting of the manuscript: Capitanio, Larcher.

Critical revision of the manuscript for important intellectual content: Capitanio, Larcher, Terrone, Antonelli, Volpe, Fiori, Furlan, Dehò,
Minervini, Serni, Porpiglia, Trevisani, Salonia, Carini, Simeone, Montorsi, Bertini.

Statistical analysis: Capitanio, Larcher.

Obtaining funding: None.

Administrative, technical, or material support: None.

Supervision: Capitanio, Larcher, Terrone, Antonelli, Volpe, Fiori, Furlan, Dehò, Minervini, Serni, Porpiglia, Trevisani, Salonia, Carini, Simeone, Montorsi, Bertini.

Other (specify): None.

Financial disclosures: Umberto Capitanio certifies that all conflicts of interest, including specific financial interests and relationships and affiliations relevant to the subject matter or materials discussed in the manuscript (eg, employment/ affiliation, grants or funding, consultancies, honoraria, stock ownership or options, expert testimony, royalties, or patents filed, received, or pending), are the following: None.

Funding/Support and role of the sponsor: None.

\section{Appendix A. Supplementary data}

Supplementary data associated with this article can be found, in the online version, at http://dx.doi.org/10.1016/j. eururo.2016.03.023.

\section{References}

[1] Capitanio U, Montorsi F. Renal cancer. Lancet 2016;387:894-906, http://dx.doi.org/10.1016/S0140-6736(15)00046-X

[2] Scosyrev E, Messing EM, Sylvester R, Campbell S, Van Poppel H. Renal function after nephron-sparing surgery versus radical nephrectomy: results from EORTC randomized trial 30904. Eur Urol 2014;65: 372-7, http://dx.doi.org/10.1016/j.eururo.2013.06.044

[3] Capitanio U, Terrone C, Antonelli A, et al. Nephron-sparing techniques independently decrease the risk of cardiovascular events relative to radical nephrectomy in patients with a T1a-T1b renal mass and normal preoperative renal function. Eur Urol 2015;67:683-9, http://dx.doi.org/10.1016/j.eururo.2014.09.027

[4] Fox CS, Matsushita K, Woodward M, etal. Associations of kidney disease measures with mortality and end-stage renal disease in individuals with and without diabetes: a meta-analysis. Lancet 2012;380:166273, http://dx.doi.org/10.1016/S0140-6736(12)61350-6

[5] Muzaale AD, Massie AB, Wang M-C, et al. Risk of end-stage renal disease following live kidney donation. JAMA 2014;311:579-86, http://dx.doi.org/10.1001/jama.2013.285141

[6] Lin W-Y, Liang F-W, Lu T-H. Risk of end-stage renal disease after cancer nephrectomy in Taiwan: a nationwide population-based study. PLoS One 2015;10:e0126965-126968, http://dx.doi.org/10. 1371/journal.pone.0126965

[7] Yap SA, Finelli A, Urbach DR, Tomlinson GA, Alibhai SMH. Partial nephrectomy for the treatment of renal cell carcinoma (RCC) and the risk of end-stage renal disease (ESRD). BJU Int 2015;115:897-906, http://dx.doi.org/10.1111/bju.12883

[8] Lane BR, Demirjian S, Derweesh IH, Riedinger CB, Fergany AF, Campbell SC. Is all chronic kidney disease created equal? Curr Opin Urol 2014; 24:127-34, http://dx.doi.org/10.1097/MOU.0000000000000029

[9] Lane BR, Demirjian S, Derweesh IH, et al. Survival and functional stability in chronic kidney disease due to surgical removal of nephrons: importance of the new baseline glomerular filtration rate. Eur Urol 2015;68:996-1003, http://dx.doi.org/10.1016/j.eururo. 2015.04.043 